ISSN electrónico: 2445-1355

DOI: http://dx.doi.org/10.14201/fj2020511726

\title{
TOALLITAS COSMÉTICAS PARA EL TRATAMIENTO DE LA HIPERHIDROSIS SIN LA PRESENCIA DE SALES DE ALUMINIO
}

\section{Cosmetic wipes for the treatment of hyperbidrosis without the presence of aluminum salts}

Irene FERNÁNDEZ SÁNCHEZ; Aránzazu ZARZUELO CASTAÑEDA

Departamento de Ciencias Farmacéuticas, Área de Farmacia y Tecnología Farmacéutica. Facultad de Farmacia

Campus Miguel de Unamuno, Licenciado Méndez Nieto, s/n. 37007 Salamanca Correo-e: fernandezschezirene@gmail.com.

RESUMEN: La hiperhidrosis es la producción de sudor anormalmente mayor a la requerida para regular la temperatura corporal. Los síntomas se manifiestan principalmente en las palmas de las manos, plantas de los pies, axilas y regiones craneofaciales. Actualmente, existen diversos tratamientos para la hiperhidrosis, siendo el tratamiento tópico con sales metálicas y, en concreto, con cloruro de aluminio hexahidratado, el más empleado por su eficacia, seguridad y facilidad de aplicación, sin embargo, algunas personas pueden experimentar reacciones de sensibilidad a las citadas sales. La formulación propuesta en este estudio proporciona una alternativa eficaz y segura para el tratamiento de la hiperhidrosis, empleando como principios activos de la formulación aceite esencial de árbol de té, vinagre de manzana, extractos de salvia y tomillo y mentol en toallitas de celulosa individualizadas. Tras probar distintas formulaciones en tres voluntarios con hiperhidrosis, las impresiones fueron satisfactorias al percibirse rápida absorción de la formulación, frescor y prolongación en el tiempo de la sequedad de la zona tratada. El resultado del estudio refleja que los preparados cosméticos con compuestos naturales pueden ser una vía de investigación satisfactoria en la búsqueda del tratamiento de la hiperhidrosis.

Ediciones Universidad de Salamanca / @@ఠ $\quad$ FarmaJournal, vol. 5, núm. 1 (2020), pp. 17-26 
Palabras clave: Hiperhidrosis; aluminio; árbol de té; vinagre de manzana; extracto de salvia; extracto de tomillo.

ABSTRACT: Hyperhidrosis is the production of sweat abnormally greater than that required to regulate body temperature. The symptoms are manifested mainly in the palms of the hands, soles of the feet, armpits and craniofacial regions. Currently, there are various treatments for hyperhidrosis, being the topical treatment with metal salts, and specifically, with aluminum chloride hexahydrate, the most used for its effectiveness, safety and ease of application, however, some people may experience sensitivity reactions to the aforementioned salts. The formulation proposed in this study provides an effective and safe alternative for the treatment of hyperhidrosis, using as essential ingredients of the formulation essential oil of tea tree, apple cider vinegar, sage and thyme extracts and menthol in individualized cellulose wipes. After testing different formulations in three volunteers with hyperhidrosis, the results were satisfactory when rapid absorption of the formulation, freshness and prolongation in time of the dryness of the treated área was perceived. The result of the study shows that cosmetic preparations with natural compounds can be a satisfactory research path in the search for the treatment of hyperhidrosis.

Keywords: Hyperhidrosis; aluminum; tea tree; apple vinager; sage extract; thyme extract.

\section{InTRODUCCIÓN}

La sudoración es un proceso fisiológico y vital para llevar a cabo la termorregulación Este mecanismo es regulado por el hipotálamo, a través de la liberación de acetilcolina en el sistema nervioso simpático, la cual se une a receptores muscarínicos de las glándulas sudoríparas ecrinas, produciendo sudor y disminuyendo la temperatura corporal (Ariel et al., 2011).

La hiperhidrosis es la producción de sudor anormalmente mayor a la requerida para regular la temperatura corporal. El diagnóstico clínico se basa en analizar si la sudoración supone una incomodidad física, social o emocional para el paciente mediante distintas escalas, como la HDSS o SF-36.

Según su etiología y localización, puede ser clasificada en:

- Primaria, la cual aparece de forma focal, idiopática, bilateral y simétrica en axilas, palmas de las manos, plantas de los pies o regiones craneofaciales, al ser las zonas donde más predominan las glándulas sudoríparas ecrinas. 
La sudoración puede aparecer de forma continua o en fases, excepto por la noche. Se inicia generalmente con la pubertad y el $40 \%$ de los casos tiene antecedentes familiares.

- Secundaria, también denominada generalizada, a pesar de que puede presentarse focalmente. Generalmente tiene lugar por la presencia de patologías concomitantes de tipo endocrino, cardiovascular, respiratorio, neurológico o infeccioso o por el uso de determinados fármacos como antidepresivos, antivirales, colinérgicos o antibióticos. A diferencia de la primaria, puede darse cuando el paciente está dormido.

A pesar de que la etiología de la hiperhidrosis primaria no se conoce con certeza, las principales propuestas sobre su origen son la predisposición genética a padecer la patología con rasgo autosómico dominante, o bien por un desorden del sistema nervioso autónomo, viéndose afectadas las vías simpáticas y parasimpáticas, lo que originaría una hiperactividad neurogénica de las glándulas sudoríparas ecrinas (Nawrocki, Cha, 2019).

La hiperhidrosis puede producir problemas dermatológicos mayores como irritaciones, descamaciones, infecciones o bromhidrosis o reducir la calidad de vida de los pacientes, pudiendo dar lugar a problemas psicológicos y emocionales como inseguridad, ansiedad o depresión, que afectan tanto a la vida personal como profesional de los pacientes (Hashmonai et al., 2017).

El tratamiento actual para esta patología puede clasificarse en clínico y quirúrgico; dentro del clínico se encuentra el tratamiento tópico, que se centra en el empleo de sales metálicas, las cuales presentan propiedades astringentes al actuar bloqueando físicamente el ducto sudoríparo por la formación de precipitados por parte de los iones de aluminio. Se consideran la primera línea de tratamiento por su disponibilidad, relación coste-efectividad y fácil uso, pero pueden producir irritación o quemazón en la zona. El más empleado para combatir la hiperhidrosis leve-moderada, sobre todo en axilas, es el cloruro de aluminio hexahidratado.

La iontoforesis, la toxina botulínica y el sistema de microondas controladas Miradry también forman parte del tratamiento tópico de la hiperhidrosis.

El tratamiento sistémico se basa en la administración de fármacos anticolinérgicos, los cuales inhiben la liberación de acetilcolina, neurotransmisor implicado en la excreción del sudor por parte de las glándulas sudoríparas ecrinas. Su inconveniente son los efectos secundarios como sequedad de boca, estreñimiento o retención urinaria.

Por último, el tratamiento quirúrgico es el de elección cuando los tratamientos anteriormente citados no dan lugar a resultados satisfactorios (Nawrocki, Cha, 2019). 


\section{2. Овјетivo}

El objetivo del estudio fue el diseño y elaboración de una formulación cosmética para tratar la hiperhidrosis primaria de carácter leve o moderado, utilizando componentes naturales impregnados en toallitas individualizadas, que proporcione una alternativa efectiva contra la hiperhidrosis especialmente en personas con sensibilidad a los metales y a sus sales, ya que se ha observado que las toallitas comercializadas a día de hoy presentan en su composición sales metálicas.

\section{Materiales y métodos}

La información de los componentes a incluir en las fórmulas magistrales fue encontrada en bases de datos de carácter científico como SciFinder, Pubmed, ScienceDirect y distribuidores de materias primas para farmacia y cosmética como Acofarma y Guinama, utilizando como criterios de búsqueda aquellos que relacionan la hiperhidrosis con los conceptos fitoterapia y aceites esenciales.

Una vez seleccionados los componentes más interesantes, se realizó una nueva búsqueda empleando como criterios de búsqueda el nombre del aceite, extracto o componente seguido de los conceptos «propiedades», «sudor» o «hiperhidrosis». Las referencias obtenidas se acotaron a los últimos veinte años.

\section{Resultados y discusión}

Con la información obtenida, se propusieron y elaboraron en el laboratorio cuatro fórmulas cosméticas de partida, recogidas en la tabla 1.

Los componentes fueron elegidos principalmente por sus propiedades astringentes, anhidróticas, antisépticas y refrescantes.

Aceite esencial de árbol de té: Empleado como uno de los ingredientes activos de la formulación, por su propiedad antimicrobiana, atribuida a su elevado contenido en hidrocarburos terpénicos, principalmente monoterpenos (terpinen-4-ol, $\Upsilon$-terpineno y a-terpineno) y sesquiterpenos (Carson et al., 2006).

Se trata de un líquido límpido, volátil, de aroma característico, insoluble en agua y miscible en etanol que no presenta efectos irritantes ni tóxicos por vía tópica y que puede ser empleado en cosmética a una concentración máxima del 2\% (Muruzábal et al., 2015).

En la formulación, su poder antimicrobiano ayuda a eliminar la flora bacteriana responsable de la descomposición del sudor y el mal olor asociado a ello. 
TABla 1. Composición cualitativa y cuantitativa de las formulaciones 1-4 propuestas

\begin{tabular}{|c|c|c|c|c|c|}
\hline \multicolumn{6}{|c|}{ Fórmulas } \\
\hline Componentes & 1 & 2 & 3 & 4 & Propiedades \\
\hline $\begin{array}{c}\text { Aceite esencial de árbol } \\
\text { de té }\end{array}$ & $1 \%$ & $1 \%$ & $1 \%$ & $1 \%$ & Antimicrobiano \\
\hline $\begin{array}{c}\text { Extracto glicólico de } \\
\text { salvia }\end{array}$ & $2 \%$ & $2 \%$ & $2 \%$ & $2 \%$ & $\begin{array}{c}\text { Astringente, antibacteriano } \\
\text { y anhidrótico }\end{array}$ \\
\hline $\begin{array}{l}\text { Extracto fluido de } \\
\text { tomillo }\end{array}$ & - & $2 \%$ & - & $2 \%$ & $\begin{array}{c}\text { Astringente, antiséptico y } \\
\text { desodorante }\end{array}$ \\
\hline Vinagre de manzana & $10 \%$ & $20 \%$ & - & - & $\begin{array}{l}\text { Antimicrobiano y } \\
\text { astringente }\end{array}$ \\
\hline Ácido tánico & - & - & $2 \%$ & $2 \%$ & $\begin{array}{l}\text { Tónico, astringente y } \\
\text { anhidrótico }\end{array}$ \\
\hline Alcohol 96\% & $20 \%$ & $20 \%$ & $20 \%$ & $20 \%$ & $\begin{array}{c}\text { Anhidrótico, antiséptico y } \\
\text { astringente }\end{array}$ \\
\hline Propilenglicol & $10 \%$ & $10 \%$ & $10 \%$ & $10 \%$ & $\begin{array}{l}\text { Solvente, bactericida y } \\
\text { protector de la piel }\end{array}$ \\
\hline Cremophor RH 40 & $5 \%$ & $5 \%$ & $5 \%$ & $5 \%$ & Solubilizante \\
\hline Agua de hamamelis & $\begin{array}{c}\operatorname{csp} 100 \\
\mathrm{ml}\end{array}$ & - & $\begin{array}{c}\operatorname{csp} 100 \\
\mathrm{ml}\end{array}$ & - & Astringente y vehículo \\
\hline Agua de rosas & - & $\begin{array}{c}\operatorname{csp} 100 \\
\mathrm{ml}\end{array}$ & - & $\begin{array}{c}\operatorname{csp} 100 \\
\mathrm{ml}\end{array}$ & Astringente y vehículo \\
\hline
\end{tabular}

Extracto glicólico de salvia: Su abundante composición en metabolitos terpénicos y en compuestos fenólicos lo dotan de propiedades astringentes, antibacterianas y anhidróticas según la Comisión E Alemana.

Estas actividades farmacológicas fueron demostradas en el estudio de Blumenthal en el 2000, donde se analizó la transpiración de ocho pacientes sobre los que se aplicó extracto de salvia tópicamente, dando lugar a una reducción significativa de la sudoración (Ortega et al., 2002).

Extracto fluido de tomillo: Empleado en la elaboración de fitocosméticos por su actividad antiséptica y desodorante, gracias a su composición en fenoles monoterpénicos (como timol, carvacrol y 1,8-cineol), flavonoides, ácidos fenólicos y taninos (Bassolé, Juliani, 2012).

Este componente fue adicionado a dos de las formulaciones de partida para verificar si los terpenos de este extracto potenciaban la permeabilidad del estrato córneo, tal y como se describe en algunos estudios (Edris, 2007). 
Vinagre de manzana: Presenta características beneficiosas para la piel, entre ellas su actividad antioxidante y antimicrobiana. Esta acción se debe a los ácidos orgánicos presentes, especialmente el ácido acético, ya que acidifican la zona donde ha sido aplicado, impidiendo el crecimiento de microorganismos. También cuenta con propiedades astringentes atribuidas a los compuestos polifenólicos presentes en el vinagre de manzana, como el ácido clorogénico (Ho et al., 2017), por lo que se incluyó como ingrediente cosmético activo de las formulaciones uno y dos.

Ácido tánico: Compuesto empleado en las formulaciones tres y cuatro por ser reconocido en cosmética por sus propiedades tónicas, astringentes y anhidróticas en hiperhidrosis. En soluciones, una concentración de ácido tánico entre 2-5\%, puede reducir la transpiración al desnaturalizar la queratina y ocluir las glándulas sudoríparas de forma breve, siendo este el motivo de inclusión en el estudio (Acofarma, 2014).

Agua de rosas: Empleada en formulación como astringente, tónico, antibacteriano y vehículo principal de formulaciones. Es utilizada en la formulación por la propiedad astringente del 2 -feniletanol y la sensación calmante y refrescante sobre la piel (Agarwal et al., 2005).

Agua de hamamelis: Al igual que la anterior, se emplea como astringente y vehículo principal de la formulación por su composición en sustancias tánicas y flavonoides (Villar del Fresno, 2001).

De las cuatro fórmulas de partida elaboradas, las dos que contenían ácido tánico fueron descartadas al dar lugar a una suspensión que impedía el impregnado de las toallitas y su posterior uso.

La búsqueda de información de preparados desodorantes y antitranspirantes sugirió adicionar a las fórmulas propuestas mentol, interesante por sus propiedades antibacterianas y refrescantes, quedando recogida en la tabla 2 la composición de las dos nuevas formulaciones propuestas.

El mentol es un alcohol secundario saturado de naturaleza terpénica que se encuentra en especies del género Mentha. Es interesante en el estudio por sus propiedades antibacterianas de amplio espectro y rubefacientes, provocando sensación de frescor y analgesia (Solans y Del Pozo, 2009).

Su mecanismo de acción consiste en activar los canales TRPM-8, sensibles a bajas temperaturas y ciertas sustancias, como el mentol, que transmiten al hipotálamo la sensación de frescor en la piel. La dosis empleada en preparados cosméticos tópicos, entre 0.1-2\% (Gillis, et al., 2014). 
I. FERNÁNDEZ SÁNCHEZ, A. ZARZUELO CASTAÑEDA

TOALLITAS COSMÉTICAS PARA EL TRATAMIENTO DE LA HIPERHIDROSIS SIN LA PRESENCIA DE SALES DE ALUMINIO

TABla 2. Composición cualitativa y cuantitativa de las formulaciones 5-6 propuestas

\begin{tabular}{|c|c|c|}
\hline \multicolumn{2}{|c|}{ Fórmulas } \\
\hline Componentes & 5 & 6 \\
\hline Aceite esencial de árbol de té & $1 \%$ & $1 \%$ \\
\hline Extracto fluido de tomillo & - & $2 \%$ \\
\hline Extracto glicólico de salvia & $2 \%$ & $2 \%$ \\
\hline Mentol & $2 \%$ & $2 \%$ \\
\hline Vinagre de manzana & $10 \%$ & $20 \%$ \\
\hline Alcohol 96\% & $20 \%$ & $20 \%$ \\
\hline Propilenglicol & $10 \%$ & $10 \%$ \\
\hline Cremophor RH 40 & $5 \%$ & $5 \%$ \\
\hline Agua de hamamelis & $\mathrm{csp} 100 \mathrm{ml}$ & - \\
\hline Agua de rosas & - & $\mathrm{csp} 100 \mathrm{ml}$ \\
\hline
\end{tabular}

Sobre las cuatro fórmulas consideradas a priori candidatas (fórmulas 1, 2, 5 y 6) preparadas en el laboratorio, y antes de impregnar las toallitas, se realizó un análisis de calidad en el cual se tuvo en cuenta color, olor, presencia de precipitado y $\mathrm{pH}$ a tiempo cero y tras quince días de almacenamiento a temperatura ambiente, manteniéndose los resultados constantes a ambos tiempos.

Una vez verificado que las soluciones eran estables, se impregnaron toallitas de celulosa de 20 x 20 con $1 \mathrm{ml}$ de cada una de las fórmulas propuestas y se cerraron mediante termosellado.

Se probó la efectividad de las formulaciones 1, 2, 5 y 6 en las palmas de la manos y plantas de los pies de tres voluntarios con hiperhidrosis moderada. Para ello previamente se lavaron y secaron la zona a tratar. Tras esto, se aplicaron las fórmulas aleatoriamente sobre las manos y pies derechos de cada voluntario y se esperó hasta que comenzasen a sudar.

A simple vista, las formulaciones 5 y 6 fueron las que dieron lugar a resultados más satisfactorios en las manos, siendo la formulación 6 la más efectiva en los tres voluntarios al absorberse antes en la piel, dejar un efecto más refrescante y agradable y prolongar más tiempo la sequedad de la zona tratada con respecto a las fórmulas 1,2 y 5. 
IMAGEN 1. Voluntario tratado en la mano derecha de la imagen con la formulación 6 y mano izquierda sin tratar.

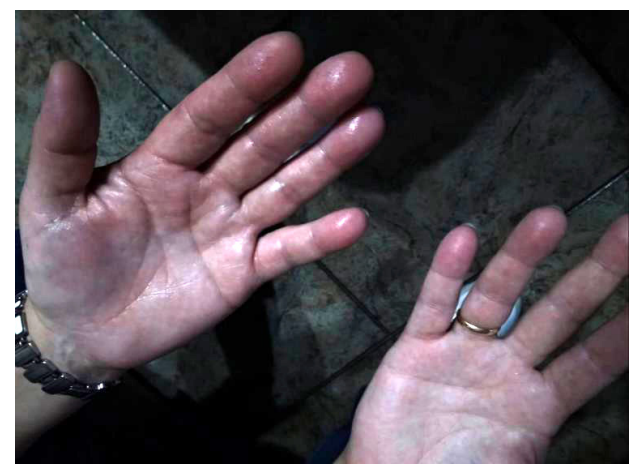

Los resultados de la aplicación de las formulaciones en los pies fueron similares a los de las manos, siendo la formulación 6, igualmente, la más efectiva.

Los mismos resultados se obtuvieron con la percepción de los usuarios, a los que se les propuso realizar una encuesta de satisfacción al finalizar el estudio. Los tres voluntarios opinaron que la fórmula más efectiva tanto en la palma de la mano como en la planta del pie era la 6, no refiriendo ningún tipo de irritación en la zona tratada. Como contrapunto coincidieron en que no les agradaba el olor de ninguna de las toallitas probadas, siendo el olor de la número seis el mejor aceptado.

\section{Conclusiones}

La hiperhidrosis es una patología que inicialmente no produce problemas físicos mayores en el paciente, pero psicológicamente supone un problema muy grave para los que la padecen, pudiendo afectar a sus actividades cotidianas.

Los preparados en forma de toallitas comercializados hasta el momento presentan ventajas, como su efectividad, seguridad, fácil transporte y aplicación en manos, pies y axilas, pero algunas personas no pueden utilizarlos por su sensibilidad o alergia a las sales metálicas. Por ese motivo se propuso este estudio, para intentar ofrecer una alternativa menos agresiva en el tratamiento de la hiperhidrosis leve o moderada, especialmente en aquellas personas que no puedan o quieran utilizar las citadas sales de aluminio.

Tras probar las formulaciones 1, 2, 5 y 6 sobre los tres voluntarios, la formulación que contiene mentol, aceite esencial de árbol de té, extractos de salvia y tomillo y vinagre de manzana fue la que dio lugar a mejores resultados, absorbiéndose antes, proporcionando más frescor y prolongando mucho más tiempo la sequedad de la zona. 
Cabe destacar que sería necesario seguir probando la citada fórmula en más voluntarios para confirmar sus efectos anhidróticos, e intentar enmascarar el olor, principalmente a vinagre de manzana, para que sea más agradable su aplicación.

Además de los distintos tratamientos disponibles para tratar los síntomas de la hiperhidrosis, es muy importante aconsejar al paciente unas medidas higiénicas adecuadas para evitar problemas dermatológicos mayores, como puede ser evitar el consumo de café, té, alcohol y tabaco utilizar ropa de tejidos transpirables, y ducharse a diario, preferiblemente con geles antisépticos.

En conclusión, el estudio mostró como los preparados cosméticos con compuestos naturales pueden ser una vía de investigación satisfactoria en la búsqueda del tratamiento de la hiperhidrosis en aquellas personas que no puedan o quieran utilizar metales.

\section{Bibliografía}

Acofarma.com [Internet]. Madrid: Acofarma; [actualizado 25 marzo 2014; citado 15 noviembre 2018]. Disponible en: https://formulasmagistrales.acofarma.com/idb/ descarga/3/f32e45b8833ba874.pdf.

Agarwal GS, Gupta A, Kapahi BK, Meena B, Thappa RK, Suri OP. Chemical composition of rose water volátiles. J. Essent. Oil Res. 2005; 17(3):265-267.

Ariel HN, Navarrete C, Montoya J. Hiperhidrosis focal primaria y uso de cloruro de aluminio: síntesis de la evidencia disponible. Dermatología Cosmética, Médica y Quirúrgica. 2011; 9(3):234-238.

Bassolé Nestor HI, Juliani RH. Essential oils in combination and their antimicrobial properties. Molecules (Basel, Switzerland). 2012; 17(4):3989-4006.

Carson CF, Hammer KA, Riley TV. Melaleuca alternifolia (tea tree) oil: a review of antimicrobial and other properties. Clin Microbiol Rev. 2006; 19(1):50-62.

Edris AE. Pharmaceutical and therapeutic potentials of essential oils and their individual volatile constituents: a review. Phytother Res. 2007; 21(4):308-323.

Gillis DJ, Weston N, House JR, Tipton MJ. Influence of repeated daily menthol exposure on human temperature regulation and perception. Physiol behav. 2015; 139:511-518.

Hashmonai M, Cameron AEP, Connery CP, Perin N, Licht PB. The Etiology of Primary Hyperhidrosis: A Systematic Review. Clin Auton Res. 2017; 27(6):379-383.

Ho CW, Lazim AM, Fazry S, Zaki UKHH, Lim SJ. Varieties, production, composition and health benefits of vinegars: A review. Food Chem. 2017; 221:1621-1630.

Muruzábal SR, Garcés HM, García LM, Pascual LL, Pérez AA, Bayona YI. Efectos secundarios de la aplicación tópica de un aceite esencial. Dermatitis alérgica de contacto a aceite de árbol de té. Anales del Sistema Sanitario de Navarra. 2015; 38(1):163-167.

Nawrocki S, Cha J, The Etiology, Diagnosis and Management of Hyperhidrosis: A Comprehensive Review. Part I. Etiology and Clinical Work-Up, J Am Acad Dermato. 2019. DOI: https://doi.org/10.1016/j.jaad.2018.12.071. 
Nawrocki S, Cha J, The Etiology, Diagnosis and Management of Hyperhidrosis: A Comprehensive Review. Part II. Therapeutic Options, J Am Acad Dermato. 2019. DOI: https://doi.org/10.1016/j.jaad.2018.11.066.

Ortega HT, Carretero AM, Villar A. Salvia. Fitoquímica, farmacología y terapéutica. Farmacia Profesional. 2002; 16(7):60-63.

Solans M, Del Pozo A. Cosmética y práctica deportiva (II). Preparados refrescantes. Offarm. 2009; 28(3):72-74.

Villar del Fresno, AM. Hammamelis virginiana. Fitoquímica, farmacología y terapéutica. Farmacia Profesional. 2001; 15(6):86-90. 